\title{
EGG SIGNAL CLASSIFICATION USING PRINCIPAL COMPONENT ANALYSIS
}

\author{
Subramanya Nayak G. ${ }^{1}$, Dr. Puttamadappa C. ${ }^{2}$ \\ ${ }^{1}$ Manipal Institute of Technology, Manipal- 576104, \\ ${ }^{2}$ Sri Jagadguru Balagangadharanatha Institute of Technology, Bangalore \\ E-mail: 'gs_nayak@rediffmail.com
}

\begin{abstract}
The method is used to register the laryngeal behavior indirectly by measuring change in the electrical impedance across the throat during speak or voice. In this Electroglottography (EGG) signal acquisition, the electrodes are made of steel. They have the form of rectangles covering an area of $10.75 \mathrm{~cm} 2$. It is designed as a ring electrode encircling each of the two other electrodes. The electrodes are mounted on a flexible band whose length is adjusted to hold the electrodes in a steady position and to still allow the subject to comfortably speak and breathe naturally. The electrodes are mounted on a small holder which is pressed against the throat by hand. A signal generator supplies an AC sinusoidal current usually ranging from $2 \mathrm{MHz}$. The RF carrier signal is amplitude modulated by the modulating speech/voice signal and the demodulated signal is extracted. The variations in the signal correspond to the vocal fold abduction/laryngeal movement. For normal and pathology conditions, the results are recorded. These values form a feature vector, which reveals information regarding pathology. Principal Component Analysis technique (PCA) is used for classification, giving successful results for the specific data set considered.
\end{abstract}

Keywords: Electroglottography, Principal Component Analysis.

\section{INTRODUCTION}

The voice pathology is very common in all over the world. In the current study the vocal fold abduction /laryngeal movement of normal and pathology patients have been recorded in terms of demodulated signals and MATLAB @6.1 supported Principal Component Analysis technique (PCA) is used for classification, giving successful results for the specific data set considered.

\section{INSTRUMENTATION}

The electrodes are made of steel. They have the form of rectangles covering an area of $10.75 \mathrm{~cm} 2$. It may be designed as a separate electrode or as a ring electrode encircling each of the two other electrodes. The electrodes are mounted on a flexible band whose length may be adjusted to hold the electrodes in a steady position and to still allow the subject to comfortably speak and breathe naturally. The electrodes are mounted on a small holder, which is pressed against the throat by hand. A signal generator supplies an AC sinusoidal current usually ranging from $300 \mathrm{KHz}$ to $5 \mathrm{MHz}$ as shown in Figure.1.

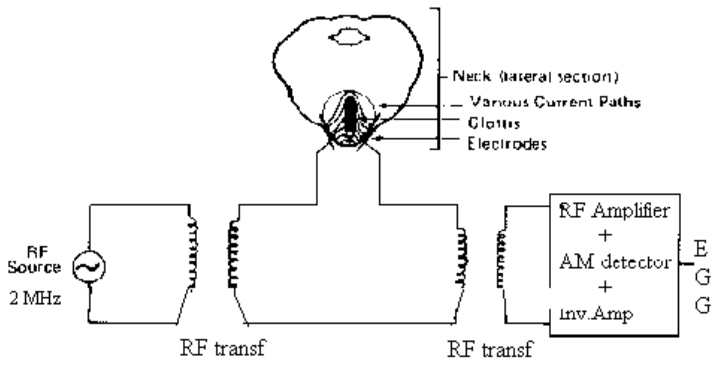

Fig. 1. Instrumentation for EGG
The frequency selected for the above test is $2 \mathrm{MHz}$. This frequency is sufficiently high, so that the current capacitancively bypasses the less conductive skin layer without the use of additional conductive paste [1]. The generator may produce constant voltage or constitute constant current

source [1]. The supplied current is different for each particular device, but is not stronger than several milliamperes. The voltage between the electrodes depends on the tissue impedance [1-3]. The power dissipation of only several microwatts occurs at the level the subject's vocal folds. An integral part of the electroglottographic signal is the varying component generated by the vertical movement of the whole larynx. Therefore, the signal of rapid movements of the vocal folds is superimposed on the signal produced by the slower movements of the other structures. Fourcin \& Abberton proposed the name Gx for the waveform of larynx movement and the name $L x$ for the vibration component. The Gx component originates, for example can be observed in swallowing, but it is caused by the vertical movement of the larynx, which is related to the voice quality setting of the raised/lowered larynx. Gx to calculate vocal fold abduction [1] The DC offset changes (Gx) can be evened out because, the effects of the varying larynx height are compensated by the use of additional electrodes or high pass filtering of the registered signal. The sensing electrode detects the current as it passes through the skin and the throat. The percentage of amplitude modulation of the received signal reflects the percentage change in 3 tissue impedance in the current's path. The output from the second RF transformer is then amplified using the above RF amplifier circuit. The output 
is demodulated using a diode detector circuit $(G x+L x)$ .The output is then amplified using a OPAMP inverting amplifier (Gx) as shown in Fig. 2. The output spectra were

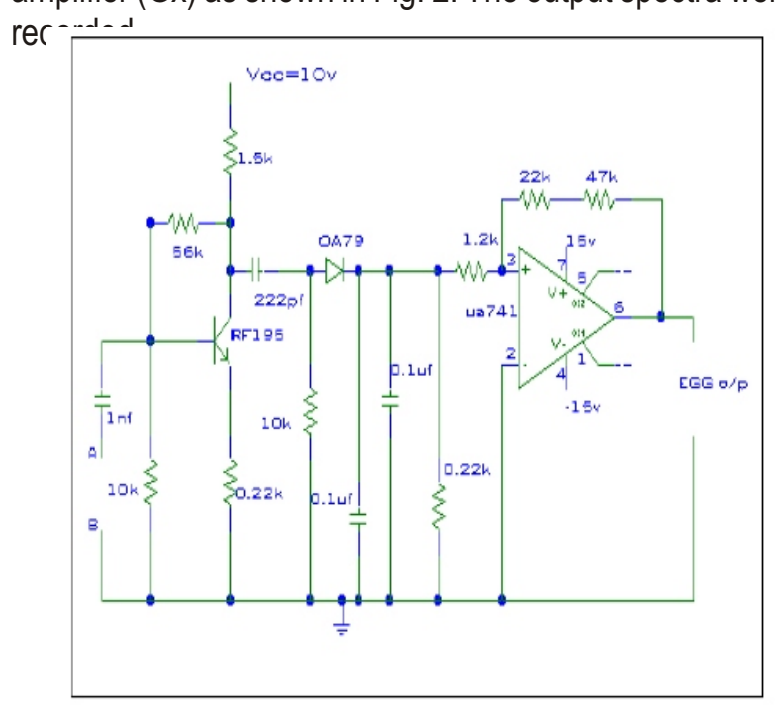

Fig. 2. RF Amplifier and Detector

\section{PCA ANALYSIS}

In the development of diagnostic or analytical methods for routine applications, only a small amount of data from a very large quantity of data is made use of from the point of view of expediency. This may lead to wrong interpretation and consequent faulty decision making, especially in clinical applications where personal judgment of the clinician may influence the decision. This kind of subjective evaluation of data can be avoided when we have enough data (e.g. a large number of spectra, each consisting of several data points) by appropriate mathematical / statistical analysis. Almost always, the enormous amount of data could be understood in terms of a much smaller number of components, called principal components or factors. This is equivalent to the situation, where any number of vectors in 3-dimentional spaces can be expressed in terms of 3 unit vectors and characteristic numbers for each vector. The $n$ number of spectra with $p$ data points each may be expressed in terms of a much smaller number of components or factors each with $p$ data points. The identification of these unique factors is known as Principal ComponentAnalysis (PCA) [4,5].

In real samples, there are usually many different variations that make up a spectrum: the constituents in the sample mixture, inter-constituent interactions, instrument variations such as detector noise, changing environmental conditions that affect absorbance, and differences in sample handling. Yet, even with all these complex changes occurring, there should be some finite number of independent variations occurring in the spectral data. Hopefully, the largest variations in the calibration set would be changes in the spectrum due to different concentrations of the constituent of the mixtures. If it were possible to calculate set of "variation spectra" that represented the changes in the intensities at all the wavelengths in the spectra, then this data could be used instead of raw spectral data for building the calibration model. These should be fewer common variations than the number of calibration spectra (in most cases), and thus, the number of calculations for the calibration equations will be reduced as well.

Presumably, the "variation spectra" could be used to reconstruct the spectrum of a sample by multiplying each one by an appropriate constant scaling factor and adding the results together until the reconstructed spectrum closely matches the sample spectrum. Obviously, each spectrum in the calibration set would have a different set of scaling constants for each variation since the concentrations of the constituent is different. Therefore, the scaling constant of each "spectrum" that must be added to reconstruct the unknown data should be related to the concentration of the constituents.

The "variation spectra" are often called "eigenvectors" (also called spectral loading, loading vectors, principal components or factors), from the methods used to calculate them. The scaling constants used to reconstruct the spectra are generally known as "scores".

Since the calculated eigenvectors came from the original calibration data, they must be somehow relating to the concentrations of the constituents that make up the samples. The same loading vectors can be used to predict "unknown" samples; thus only difference between spectra of samples with different constituent concentrations is the fraction of each loading added (scores).

\section{A. Signal Preprocessing}

In the present study, we have used MATLAB @6.1 software tool is used to carryout smoothing, mathematical and statistical analysis.

\section{i) Smoothing}

Highly noisy spectrum can be smoothened to a great extent using various smoothing functions. These include Fourier-domain smoothing, binomial smoothing etc. Fourier transforming the data, applying a filter function and then inverse Fourier transforming the data, accomplishes Fourier smoothing.

\section{CLASSIFICATION}

This method can classify samples into well-defined groups or categories based on a training set of similar samples without prior knowledge of the actual composition of group of training samples. The aim of this analysis is to 
identify unknown sample. The spectrum of sample is compared against the model to determine if it matches the training data for the model [6]. If the training set was constructed from spectra of samples that were of known quality, the model can accurately predict if the sample is of same quality by matching the spectrum and giving a "YES" or "NO" answer.

\section{A. Classification Parameters}

\section{i) Eigenvectors And Scores}

Eigenvectors are the spectral equivalents of principle components of the sample and scores corresponding to contribution of each principle component to a given sample. Multiplying the eigenvectors with the scores for that sample and adding the product for all scores can reconstruct each sample spectrum.

\section{ii) Residual Errors Or Spectral Residual}

When each sample is predicted, a set of scores is found that best fits the model loading vectors to the sample spectrum. By using the calculated scores and calibration loading vectors, a new model reconstructed spectrum can be calculated. This new spectrum is what the PCA model thinks the sample spectrum look like. The residual errors or spectral residual is the difference between this spectrum and the actual prediction spectrum.

\section{iii) Mahalanobis Distance}

It is very sensitive to inter variable changes in calibration data. The distance is measured in terms of standard deviations from the mean of the training samples The values give a statistical measure of how well the spectrum of unknown sample matches the original training spectra. Typical discrimination model is as shown in Figure 3.

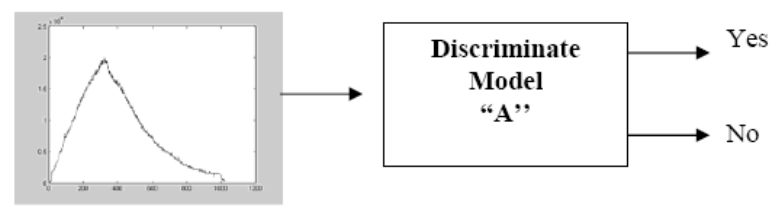

Fig. 3. Typical Discrimination Model

In PCA analysis, twenty spectra each from certified normal and pathology samples (details of spectra used are listed in table 1) were combined to see the best approach to prepare calibration sets in the two classes. It is seen that the eigenvalues decrease very rapidly and are almost zero after 7 to 8 factors, and about $99 \%$ of total spectral contribution come from these factors only as shown in Table 2. From the Table 2 and Figure 4, it can be seen that the eigenvalues decrease very rapidly and are almost zero after eight factors and also $100 \%$ of the total spectral contribution come from these eight factors. This can be further confirmed by using an appropriate number of factors from the model set and regenerating the spectrum of any sample. The difference between the observed and regenerated spectrum, expressed as residual errors squared sum can be used as a measure of desired number of factors, as well accuracy of the model. In the present analysis it is found that four factors contributed to about $98 \%$ of total variance, and these four factors completely describe the spectra. The higher factors were found only to account for variations in day-to-day runs, noise etc., and did not improve sum of squared spectral residuals, or other parameters like average predicted Mahalanobis distance [7]. All final calculations were thus carried out with using only four factors. In this case, the statistical parameters like spectral residuals, Mahalanobis distances etc. are used for discrimination between normal and pathology cases. (a)

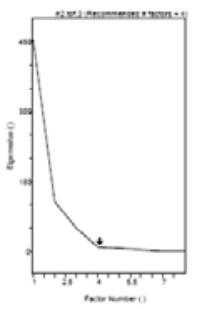

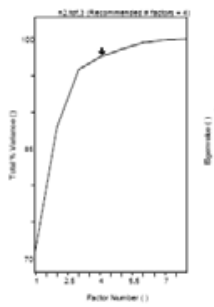

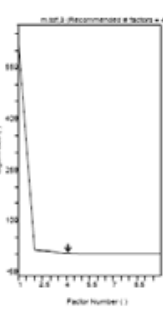

(b)

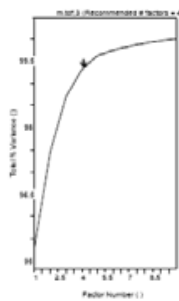

Fig. 4. PCA Eigen values and total $\%$ variance for a model set of (a) 20 normal spectra

(b) 20 pathology spectra.

Table 1. Spectral Details

\begin{tabular}{|c|c|c|c|c|}
\hline $\begin{array}{c}\text { Spectral } \\
\text { No }\end{array}$ & $\begin{array}{c}\text { Sample } \\
\text { Type }\end{array}$ & Mean age & Histopathology & Signal \\
\hline $1-20$ & $\begin{array}{c}\text { Normal } \\
\text { Standard Set }\end{array}$ & $48 \pm 6.1$ & $\begin{array}{c}\text { Uninfected area - } \\
\text { Normal }\end{array}$ & - \\
\hline $21-40$ & $\begin{array}{c}\text { Pathology } \\
\text { Standard set }\end{array}$ & $48 \pm 6.1$ & $\begin{array}{c}\text { Laryngeal movement / } \\
\text { vocal fold abduction }\end{array}$ & - \\
\hline $41-80$ & $\begin{array}{c}\text { Normal Test } \\
\text { Set }\end{array}$ & $44 \pm 7.5$ & $\begin{array}{c}\text { Uninfected area - } \\
\text { Normal }\end{array}$ & Normal \\
\hline $81-117$ & $\begin{array}{c}\text { Pathology } \\
\text { Test Set }\end{array}$ & $44 \pm 7.5$ & $\begin{array}{l}\text { Laryngeal movement/ } \\
\text { vocal fold abduction }\end{array}$ & Pathology \\
\hline
\end{tabular}

Table 2. Factor number with corresponding eigen values and total percentage variance for 20 normal and 20 pathology calibration spectra

\begin{tabular}{|c|c|c|}
\hline Factor Number & Eigen value & Total \% Variance \\
\hline 1 & 453.980101 & 71.1774449 \\
\hline 2 & 107.09752 & 88.0199317 \\
\hline 3 & 50.1468976 & 95.841953 \\
\hline 4 & 10.7986678 & 97.5396094 \\
\hline 5 & 7.3732443 & 98.7601175 \\
\hline 6 & 5.16731475 & 99.5704086 \\
\hline 7 & 2.36394707 & 99.8509443 \\
\hline 8 & 0.01421524 & 100 \\
\hline
\end{tabular}


The Mahalanobis distance is normally expressed in units of standard deviation. For classification of oral tissues, we have employed the Mahalanobis distance (Mdistance) and spectral residual (the residual error squared sum) as the criteria. The M-distance can be represented by:

$\mathrm{D} 2=(\mathrm{S}$ test $) \mathrm{M}-1$ (S test $) 1$,

Where $S$ test is the vector of scores and sum of squared spectral residuals for a given test sample, and $M$ is given by $S \phi S /(n-1)$, where $S$ contains the corresponding parameters for the calibration set ( $n$ standards).

Since the Mahalanobis distance is a standard deviation, a distance of $॥ 2$ for a sample corresponds to a $5 \%$ probability of the sample belonging to the standard set, and higher distances will have still less probability. There are two main advantages in using D2 as a discriminating parameter. As seen from the equation, D2 explicitly accounts for any correlations between the variables, namely scores of factors. By fixing an upper limit for inclusion in any class represented by the standard calibration set for that class, we can possibly achieve any desired level of discrimination for staging.

We have made match mismatch tables of calibration set as well as test set samples (normal and malignant spectra) by comparing these with the normal calibration set considering the Mahalanobis distance (M-distance) of »3. The results are listed in tables 3, 4, 5 and 6 .

Table 3. Retrospective test of normal calibration set samples against calibration set of normal samples. Mean M distance for normal calibrated set is $1.0602 \pm 0.51$ and Mean Spectral residual is $0.7569 \pm 0.028$. Acceptance value is fixed to twice the mean $\mathrm{M}$ distance of normal calibration set.

\begin{tabular}{|c|c|c|c|c|}
\hline $\begin{array}{c}\text { Spectral } \\
\text { number }\end{array}$ & Match & M. Distance & Limit test ${ }^{* * *}$ & $\begin{array}{c}\text { Spectral } \\
\text { residual }\end{array}$ \\
\hline 1 & YES & 0.7692 & PASS (PPP) & 0.5074 \\
\hline 2 & YES & 1.0833 & PASS (PPP) & 0.9164 \\
\hline 3 & YES & 0.8911 & PASS (PPP) & 0.7391 \\
\hline 4 & YES & 1.0007 & PASS (PPP) & 0.1847 \\
\hline 5 & YES & 0.8405 & PASS (PPP) & 0.5694 \\
\hline 6 & YES & 1.7137 & PASS (PPP) & 1.5398 \\
\hline 7 & YES & 0.8633 & PASS (PPP) & 0.2917 \\
\hline 8 & YES & 1.3845 & PASS (PPP) & 0.2797 \\
\hline 9 & YES & 1.0032 & PASS (PPP) & 0.5828 \\
\hline 10 & YES & 1.6049 & PASS (PPP) & 1.3779 \\
\hline 11 & YES & 1.7517 & PASS (PPP) & 1.5069 \\
\hline 12 & YES & 1.7636 & PASS (PP?) & 1.3078 \\
\hline 13 & YES & 0.7862 & PASS (PPP) & 0.9804 \\
\hline 14 & YES & 0.5342 & PASS (PPP) & 0.7848 \\
\hline 15 & YES & 0.5356 & PASS (PPP) & 0.6027 \\
\hline 16 & YES & 0.7404 & PASS (PPP) & 0.6673 \\
\hline 17 & YES & 1.2598 & PASS (PPP) & 0.5892 \\
\hline 18 & YES & 0.8114 & PASS (PPP) & 0.5102 \\
\hline 19 & YES & 1.0734 & PASS (PPP) & 0.9164 \\
\hline 20 & YES & 1.2451 & PASS (PPP) & 0.5988 \\
\hline
\end{tabular}

Table 4. Retrospective test of pathology calibration set samples against calibration set of normal samples. Mean $M$ distance for normal calibrated set is $1.0602 \pm 0.51$ and Mean Spectral residual is

$0.7569 \pm 0.028$. Acceptance value is fixed to twice the mean $\mathrm{M}$ distance of normal calibration set.

\begin{tabular}{|c|c|c|c|c|}
\hline $\begin{array}{c}\text { Spectral } \\
\text { no }\end{array}$ & Match & M distance & Limit test** & $\begin{array}{c}\text { Spectral } \\
\text { residual }\end{array}$ \\
\hline 21 & NO & 7.8591 & FAIL (FFF) & 5.0169 \\
\hline 22 & NO & 8.5018 & FAIL (FFF) & 5.3739 \\
\hline 23 & NO & 7.3529 & FAIL (FFF) & 4.6739 \\
\hline 24 & NO & 4.9425 & FAIL (FFF) & 3.3747 \\
\hline 25 & NO & 8.5206 & FAIL (FFF) & 5.4821 \\
\hline 26 & NO & 7.8294 & FAIL (FFF) & 5.0665 \\
\hline 27 & NO & 5.4197 & FAIL (FFF) & 3.6616 \\
\hline 28 & NO & 5.5963 & FAIL (FFF) & 3.6608 \\
\hline 29 & NO & 5.1404 & FAIL (FFF) & 3.6272 \\
\hline 30 & NO & 4.7382 & FAIL (FFF) & 5.0196 \\
\hline 31 & NO & 5.4372 & FAIL (FFF) & 3.5981 \\
\hline 32 & NO & 10.099 & FAIL (FFF) & 6.3894 \\
\hline 33 & NO & 4.9682 & FAIL (FFF) & 2.4129 \\
\hline 34 & NO & 4.9958 & FAIL (FFF) & 2.4899 \\
\hline 35 & NO & 16.0364 & FAIL (FFF) & 9.5869 \\
\hline 36 & NO & 4.5523 & FAIL (FFF) & 2.5229 \\
\hline 37 & NO & 4.7859 & FAIL (FFF) & 2.8999 \\
\hline 38 & NO & 4.6783 & FAIL (FFF) & 5.0518 \\
\hline 39 & NO & 7.8393 & FAIL (FFF) & 5.0665 \\
\hline 40 & NO & 8.9971 & FAIL (FFF) & 2.5192 \\
\hline
\end{tabular}

Table 5. PCA of test normal samples against calibrated set of standard normal samples. Mean M distance for normal calibrated set is 1.0161 and Mean Spectral residual is 0.7112 . Acceptance value of $\mathrm{M}$ - distance $=3.0$.

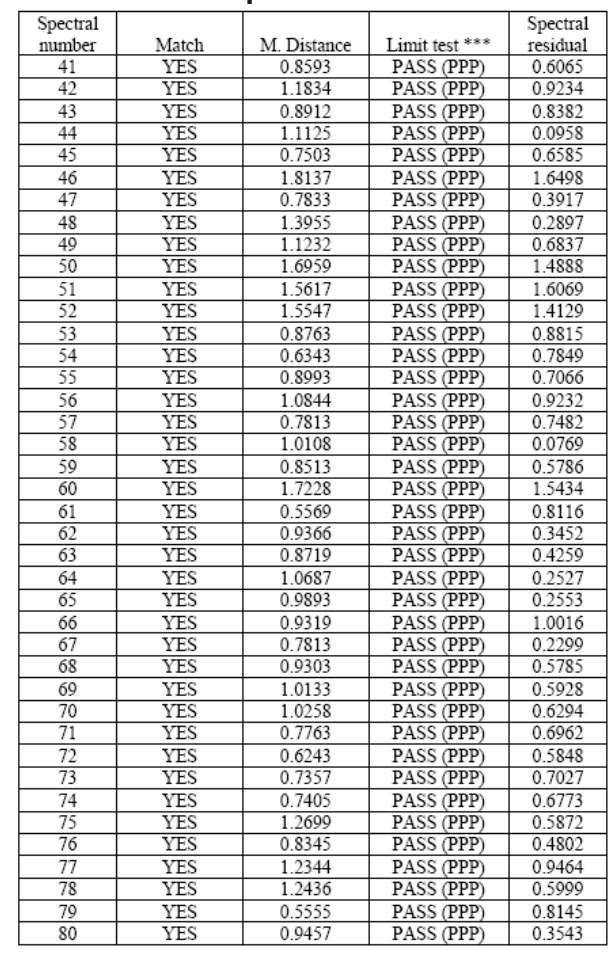

\section{DISCUSSION}

In PCA analysis, we have used twenty spectra each from certified normal and malignant oral tissue samples and calibration set is built. As mentioned earlier, we have 
used four factors for all final calculations and M-distance and spectral residual as discrimination parameters. Figure 5 , shows a plot of the M-distance against residual errors squared sum for a new set of 77 samples ( 40 normal +37 pathology), compared to a standard set of normal spectra. It is clearly seen from the plot that all samples diagnosed as normal by pathological examination in the new set fall in the lower left-hand corner of the plot. If we take a Mdistance of 1 as acceptance, then almost all the samples classified as normal samples fall within 3 times this value, while all samples classified as pathology lie far outside. The specificity and sensitivity of this technique is thus quite good, $100 \%$ and $83.8 \%$ respectively (shown in table 7 ). A closer observation of Figure 5 shows a very small number of samples outside the acceptable range of either normal or pathology species. i.e. the overlap between two sets are negligible up to mean +-2 standard deviations, which shows the probability of samples being in the respective clusters to be about $97 \%$ and finding them out of the cluster is less than $3 \%$. All the pathology samples have a Mdistance $\gg>2$, indicating the probability of their belonging to the normal group, practically zero. All the normal samples have a M-distance much lower than 2, showing the probability of these being out of the group negligible. Once the validity of the standard calibration sets was established, we carried out a limited test on the predictive value of the method. For this 77 additional spectra (40 normal and 37 pathology spectra) were predicted with the standard calibration set prepared earlier.

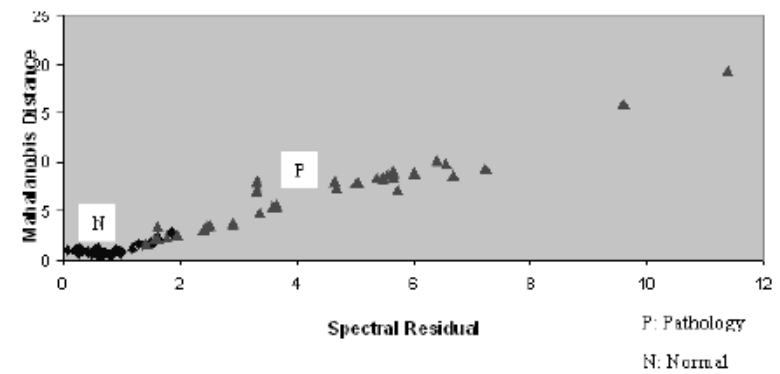

Fig. 5. Plot of spectral residual v/s M.distance- normal and pathology spectra against normal calibration set

For better discrimination of normal and pathology spectra, we have used match mismatch criteria by comparing calibration set as well as test set samples (normal and pathology spectra) with the normal calibration set considering the Mahalanobis distance (M-distance) of »3. According to this criterion, all spectra that fall within the limits are labeled as 'match' and others are labeled as 'no match'. When a set of 20 spectra of normal samples is used as calibration standard, all normal spectra of the calibration set were shown 'match' and all pathology calibration set spectra were shown 'no match' as shown in Table 3 and 4 respectively. In this case all the normal spectra are tested retrospectively by rotating out each spectrum from the calibration set, while all the pathology spectra are tested prospectively.

PCA of this region was then repeated in the prediction mode for testing match or mismatch of test samples with the normal calibration set. As expected when the set of 40 spectra of normal samples were shown 'match' and 31 out of 37 pathology spectra were shown 'no match' as shown in Table 5 and 6. Six pathology test spectra which shown match when normal calibration set was used for prediction of 37 pathology spectra may be due to the recording of the spectra from normal site of the pathology tissue. From the tables it can be seen that the results are very satisfactory and the PCA using match mismatch can be used for the discrimination between normal and pathology cases.

We have also plotted M-distance versus sample number for 117 spectra $(20+40$ normal and $20+37$ pathology) as shown in Figure 6.

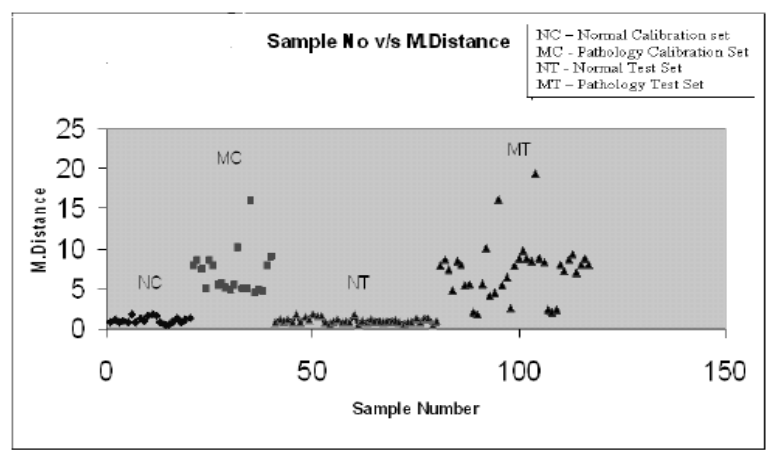

Fig. 6. Classification of 117 spectra ( $20+40$ normal, $20+37$ pathology). Sample number against $M$. distance for normal and pathology calibration and test spectra

\section{Table 6. PCA of test pathology samples against calibrated set of standard normal samples. Mean M distance for normal calibrated set is 1.0161 and Mean Spectral residual is $\mathbf{0 . 7 1 1 2}$. Acceptance value of $\mathrm{M}$ - distance $=3.0$}

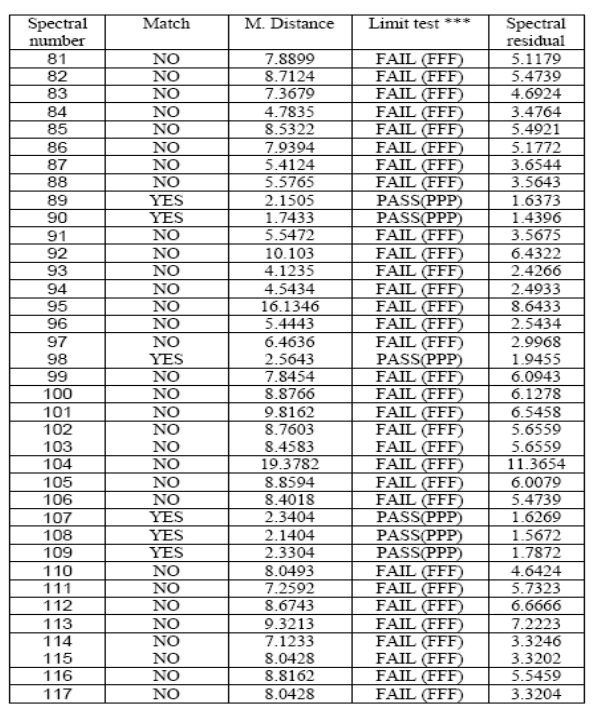


Table 7. Performance of PCA (77 test signals)

\begin{tabular}{|c|c|c|c|}
\hline Classifier & Specificity( \%) & Sensitivity( \%) & Accuracy (\%) \\
\hline PCA & 100 & 83.8 & 92.2 \\
\hline
\end{tabular}

As seen from the performance tables of PCA analysis, it is found that the sensitivity is $83.8 \%$ in case of PCA. PCA analysis play important role when biochemical composition of subject is considered [8].

In case of pathology test spectra used for prediction against normal calibration set, it is seen that there were few pathology spectra, which were classified, as normal. This deviation may be due to the recording of the spectra from normal site of patient.

\section{ACKNOWLEDGMENT}

We are thankful to Prof. Jagdish Nayak for his valuable suggestions during PCAanalysis.

\section{REFERENCES}

[1] G.Subramanya Nayak, Jagdish Nayak, 2006,, Electroglottographic Signal Acquisition and Neural Network based Classification for Pathology, IFMBE Proceedings, Vol 15,pp 54-57.

[2] Krishnamurthy, Childers, 1986, Two Channel Speech Analysis. IEEE Transactions on Acoustics, Speech and Signal Processing, ASSP-34: pp.730-743.
[3] Robert F.Orlikoff, 1991, Assessment of Dynamics of vocal Fold contact From the Electroglottogram, Journal of Speech and Hearing Reaserch, 34:1066 -1072 .

[4] Rudra Pratap, Getting started with MATLAB5, Oxford University Press, 2000

[5] William Palm J, Introduction to MATLAB 6 for Engineers.

[6] B.K.Manjunath, J.Kurien, C.Muralikrishna, V.B.Kartha, 2006, Autofluorescence of Oral tissue for optical pathology in Oral malignancy, Journal of Photochemestry and Photobiology Biology, 73, pp. 49-58.

[7] Mahalanobis, P.C., Proc. Natl. Inst. Sci. India, 1936, 2,49 .

[8] G.S.Nayak, Sudha Kamath et al. 2006, Principal Component Analysis and Artificial Neural Network Analysis of oral Tissue Fluorescence Spectra: Classification of Normal,Premelignant and Malignant Pathological Condition, Biopolymers,82:152-166. 